\title{
A positivist criticism of positivist growth theory
}

Esteban Céspedes (estebancespedes@aol.com) Instituto de Filosofía, Universidad de Valparaíso (Valparaíso, Chile) https://orcid.org/0000-0002-5329-5434

\begin{abstract}
The notion of growth is one of the most studied notions within economic theory and, traditionally, it is accounted for based on a positivist thesis according to which assumptions are not relevant, if economic models have acceptable predictive power. Following this view, it does not matter whether assumptions are realistic or not. Arguments against this principle may involve a defence of the realistic assumptions over highly idealized or false ones. This article aims in a different direction. Instead of demanding more realism, we can accept the spirit of the mentioned thesis, but, instead, criticize the circularity that may arise by combining different assumptions that are necessary for the explanation of economic growth in mainstream economics. Such a circularity is a key aspect of the well-known problem of providing microfoundations for macroeconomic properties. It is here suggested that the notion of emergence could be appropriate to arrive at a better understanding of growth, clarifying the issues related to circularity, but without totally rejecting the usefulness of unrealistic assumptions.
\end{abstract}

Key words: growth, positive economics, context-dependence, emergence.

\section{Introduction}

According to traditional positive economics, the realism of the assumptions that sustain economic models does not matter; what matters is the model's predictive power. If a model generates empirically adequate predictions, it can be accepted, even based on implausible assumptions. According to different arguments, in favour of and against the project of positive economics, it is assumed that theories are postulated in relative independence of other theories in the field. Usually, each proposed theory is defended, examined, and criticized in its own light. But what occurs when we consider related theories simultaneously? (One anonymous referee motivated me to consider this matter, highlighting its relation to the present work.)

I will focus on the mainstream, general model of macroeconomic growth considering traditional, microeconomic price theory, granting that both can be rendered as plausible by positivist standards if evaluated within their respective contexts. As is well known, a circularity problem may appear. On the one hand it is assumed, according to the general neoclassical model of economic growth, that the values of capital and labour determine, as inputs, the value of production. But, on the other hand, price theory implies that production determines the value of capital and labour. This explanatory circularity seems to raise a particular issue for positive economics, which is, in a certain sense, orthogonal to concerns over the irrelevance of assumptions, I will argue, mainly because it only arises when trying to form a coherent picture of a complex, interrelated set of social phenomena. 
If the mentioned circularity is vicious, it would be seriously problematic for the field. At a minimum, it would mean that there are core elements of economic phenomena that mainstream economists are not explaining, regardless of the empirical adequacy of the individual theories in their specific domains. I propose a different diagnose. I will show that the notions of epistemic context and contextual emergence, taken together, help us make sense of what is going on with the apparent circularity in an epistemically fruitful manner.

Based on the notion of an epistemic context, I will argue that specific explanatory models must be understood as operating within projects of inquiry, which may include not only detailed descriptions of phenomena, but also normative and nominative commitments. Epistemic contexts may be compared to Kuhnian paradigms; they are based on sociological grounds and are constituted by habits, which clearly have normative aspects. A crucial difference between both notions, however, is that epistemic contexts should be conceived as more general. Every Kuhnian paradigm is an epistemic context, but not every epistemic context is a paradigm. Anyway, I prefer not to engage in a detailed comparison now.

According to the notion of contextual emergence, I hold that emergent properties are reducible in some epistemic contexts and irreducible in others. Additionally, there must be a context in which it is possible to explain the new properties as correlates of abrupt changes in complexity at the underlying level of the system that is being investigated.

Adopting this proposal, as I would like to show, the circularity between macroeconomic growth theory and microeconomic price theory can be understood as an instance of the idea that each theory may just be part of a more general epistemic context. From a broader perspective, they may constitute the characterization of emergent, multi-level economic phenomena. At some stages of such a characterization, the action of individual agents could be seen as aggregating up into processes that ground a notion of economic growth that is recognizable at the collective level. But this need not be considered problematic, as long as it is acknowledged that every research project is always context-dependent. Contexts that are mainly focused on aggregation may turn out to be extremely reductionistic and could involve normative aspects that are fundamentally associated with social control and economic policy. These are contextual features that should be made explicit. When we continue to take multiple contexts into account, we gain a richer set of insights into the core phenomena.

As mentioned above, I would like to focus on the notion of economic growth to show how the irrelevance thesis grounds its neoclassical interpretation. This is the topic of the third section. Before doing this, the main theses of neoclassical positive economics are introduced in Section 2. Section 3 is about economic growth. In Section 4, I consider the problem of circularity associated with the general form of neoclassical growth models. Then, in the fifth section, it is argued in which sense the issue can be considered as a problem of vicious circularity rather than as an unproblematic one. I try to show that it is a problem for a positivist economic perspective (or at least for its reductionistic versions), a perspective that neoclassical theory is supposed to defend. As explained, the problem of circularity is deeply connected to the well-known problem of the microfoundations of macroeconomic properties (Section 5). According to the contextual notion of emergent property that I propose, we can express, for instance, how neoclassical models of growth, constructed based on unrealistic assumptions, may be compatible with broader perspectives. Some of those assumptions are pointed out in Section 6 and considered under a proposal on emergence. Finally, 
in Section 7, I suggest how the problem of the microfoundations can be accounted for based on a contextualist notion of emergence, which seems to be fully compatible with a non-reductionist branch of economic positivism.

\section{Positive economics and the irrelevance of assumptions}

In this section, I will focus on the importance of positivist principles within economic theory to prepare the way for a discussion on two interrelated, conceptual tensions in philosophy of economics (and, perhaps, within economic theory in general) that have not been clarified satisfactorily yet. The first is the tension between the poorly understood notion of economic growth and the idea of positive economics; the second, between macroeconomics and microeconomics. It seems impossible to propose a general way of solving the second tension without considering the first.

In this article, the neoclassical notion of economic growth will be one of the main points of focus, considering that it has played a crucial role supporting economic positivism. The importance of economic growth and of positive economics is going to be acknowledged. In general, economic positivism provides methodological foundations that support economics as a scientific discipline. No scientific theory can be successful if there are unclarities about its empirical contrastability and positivism is a way that contributes to achieving and maintaining that success. And the concept of economic growth is a great example of how positivist economic theory can be very successful in some respects but still unsatisfactory regarding others. Of course, this is no reason to reject either economic positivism or the notion of economic growth. But it is a good reason to criticize both. And if we want to secure empirical adequacy of economic theory, there are good reasons to criticize economic positivism from a positivist perspective.

As mentioned above, there is a tension between microeconomics and macroeconomics. The reasons for this tension being diverse, economic growth represents one of the central points of friction. Do economies grow as firms? If they do not, on what depends on such a difference? We might be tempted to criticize the distinction between the macro and the micro in economics. But there are good reasons to maintain it. After all, the phenomena studied by microeconomics are distinct from macroeconomic phenomena. Both fields are focused on different types of phenomena, which exhibit different functions and structures. A more interesting criticism is the one about how the micro and the macro interact. And such a criticism can also maintain a positivist spirit.

The traditional distinction between the notions of positive and normative economics can be traced back to the work of John Neville Keynes (The scope and method of political economy), who proposed it emphasizing its importance for the avoidance of misconceptions. These notions may be characterized as follows:

(2.1) Positive economics. A body of systematized economic knowledge can be considered as positive economics if it is concerned with economic facts.

(2.2) Normative economics. A body of systematized economic knowledge can be considered as normative economics if it is concerned with rules and means to achieve certain economic ends.

There are, of course, many kinds of interactions between these both fields. On the one hand, when economists and politicians want to find rules of action to solve economic issues, they must consider 
economic facts carefully. On the other hand, the ways in which economists describe economic processes may be influenced by the relevance of the social problems to which they might want to apply those descriptions. However, it is commonly accepted that the influence of positive economics in normative economics is (or should be) more substantial than the influence in the opposite direction. This can be supported by a well-known strategy of Milton Friedman's account on positive economics (The methodology of positive economics). The strategy is expressed in the following thesis:

(2.3) Irrelevance of assumptions. Positive economic theories should not be evaluated regarding their assumptions but about their predictions.

According to Friedman, economic theories should be judged considering a class of phenomena that needs explanation. Good theories are the ones that have great predictive power for a given class of phenomena, even if they are based on unrealistic assumptions. Factual evidence, according to this perspective, is characterized in terms of a correspondence between predictions and experience. This is crucial, not only to understand the distinction between positive and normative economics, but also to understand in which sense positive economics is considered as more fundamental than normative economics: we should prefer theories of high predictive power as means to achieve our economic ends. And supposedly, these economic ends do not determine what a good theory is. Of course, the evaluation of an economic theory should depend on epistemic and methodological criteria, which, as such, are considered as means to epistemic ends. But this does not imply, following the traditional positivistic account, that economic ends determine such evaluations or criteria.

Friedman's argument to defend the irrelevance thesis (2.3) could be schematized as follows (Hausman Mindless or mindful economics):

\section{(2.4) Irrelevance argument}

a) The aim of economic theories is to explain or predict relevant economic phenomena and they should be evaluated considering how they satisfy this aim (economic positivism).

b) The only relevant phenomena that economic theory should explain or predict are choices and consequences of choices, particularly for market quantities.

c) Only data about choices and consequences of choices are relevant for the evaluation of an economic theory.

d) If an economic theory provides, on the basis of unrealistic assumptions, correct predictions and explanations about choices and consequences of choices, it could still be considered a good theory (i.e. a theory that satisfies its role).

Note that the irrelevance of assumptions depends, according to Friedman's story, on a methodological restriction about how to approach phenomena (a focus on prediction and explanation) and on a restriction about what are the (relevant) phenomena. These restrictions characterize what we may call reductive economic positivism:

(2.5) Reductive economic positivism. The only aim of economics is to explain or predict microeconomic phenomena, understanding prediction and explanation in terms of logical deduction (or other kind of inference) from fundamental economic facts. 
What is positivism in economics is as unclear as what is positivism in general. Many kinds of positivist perspectives have been defended since Laplace and Comte, leading sometimes to misunderstood controversies. Maybe the main problem that positivism has faced in its different forms is seeking an appropriate account about the relations between experience and the rational principles with which we organize it. One of the best attempts to tackle this issue systematically was made by the logical positivists, also called logical empiricists (Carnap Der logische Aufbau der Welt, Neurath Soziologie im Physikalismus). I will not focus on the differences between the terms "logical positivism" and "logical empiricism". Although both are usually acceptable to name the same school, distinctions might be important for a historical and deeper study of the perspectives defended by its members (Uebel "Logical Positivism"-“Logical Empiricism"). Anyway, notably, their principles demanded theories to be not only testable with experience, but also logically rigorous, conceptually clear, and compatible with scientific unity. While the program of logical positivism has suffered severe criticisms and non-empiricist, alternative epistemological approaches have been developed very plausibly, it is still acceptable to think that the mentioned criteria should be, as such, considered as satisfiable by any good empirical theory in general. As alternatives, we may think of falsificationism, historicism, constructivism, or critical theory. But a detailed comparison between them is far beyond the scope of the present work, even if such a task is crucial for a complete epistemological account of economics. I do not focus here on positivism under the supposition that it is the best of those alternatives, but rather because I am interested in the positivist foundations of positivist economics. On this basis, we can formulate positivism (regarding theories) like this:

(2.6) Positivism. Any empirical theory should satisfy the following conditions.

2.6.1. It must be testable with experience, observation, and measurements, e.g., through methods of corroboration and refutation.

2.6.2. It must be formally expressible based on logical principles, including axioms and rules of inference, but not restricted to any logic.

2.6.3. Its models must be constituted as conceptually clear and empirically plausible interpretations, associated with well characterized ontologies.

2.6.4. It must be embedded in a coherent and unified scientific framework.

Some comments on the conditions of this definition might be convenient. First, avoiding the restriction to a particular logic in 2.6.2, we can consider theories and models as open formal systems and not only as deductively closed systems. Perhaps one of the main problems of 20th century logical positivism was its restriction associated with classical logic. Regarding condition 2.6.3, note that there is another point of dissimilarity between this formulation of positivism and logical positivism as it is classically understood, i.e., as being opposed to metaphysical thinking. However, as clearly pointed out by Herbert Feigl, "the positivistic critique of metaphysics is primarily an attack upon confusions of meanings and is not intended as a wholesale repudiation of what has been presented under that label. In point of fact, 'metaphysics' has been used in such a wide variety of ways that here also a little logical analysis of meanings is indispensable" (Feigl 1943:384). And having unification in mind (2.6.4), the kind of coherence demanded here must not strictly be logical consistency but could be some sort of pragmatic compatibility (Chang Is pluralism compatible with scientific realism?). In this sense, the proposal might be extended, for the case of the social sciences, to seek also cultural, political, ecological, and ethical coherence. 
Now, the question that should be asked is whether neoclassical economic positivism adjusts to these general positivist criteria. As shown in the present work, it does not. The main reason is that it does not seem to satisfy conditions 2.6.3 and 2.6.4. This should become clear later. For my purposes, the following condition for economic positivism should be considered as sufficiently close to the neoclassical school:

(2.7) The main aim of economics is to explain and predict economic phenomena.

Note the openness of this thesis in contrast to reductive economic positivism (2.5). As should seem clear, this characterization of economic positivism is closer to John Neville Keynes' approach than to Friedman's argument. This point will be crucial later when I address the problems of circularity and microfoundations. Another important point is this one: even if positivism is sometimes understood in terms of what is expressed by 2.7, this characterization is just an emphasis on condition 2.6.1. Thus, it is a weaker and less complete version than 2.6.

We should say more about the concept of assumption. Friedman considers it mainly in a logical way, in the sense that assumptions can be seen as antecedents, i.e., as parts of hypothetical statements that, within a given language, play a certain role depending on the consequent statements associated with them by implication. This way of understanding the notion of assumption allows Friedman to support, in a logical sense, the irrelevance about whether an assumption is realistic or not. Just as the falsehood of a conditional does not logically follow from the falsehood of its antecedent, a theory cannot be discarded just because it rests on unrealistic assumptions.

Friedman was particularly focused on economic policy and his thesis on the irrelevance of assumptions can be well illustrated considering policymaking. For example, should we oppose minimum wage laws, as he did? (Friedman Value judgments in economics) What are the consequences of such kind of policy on unemployment? According to a simple supply and demand model based on perfect competition, it may reduce unemployment, some argue. The simple model may be unrealistic, but, following Friedman, it should not be tested in this regard. We should test it regarding its implications, among which we may consider possible impacts on employment.

Now, what does it mean that assumptions can be unrealistic? It is well known that Friedman did not use the word "unrealistic" in a clear and unambiguous manner. Ernest Nagel (Assumptions in economic theory) distinguished three different senses that are relevant to understand Friedman's defence of the irrelevance of assumptions. An assumption may be rendered as unrealistic: a) because it fails to describe some object exhaustively; b) because it is highly improbable on the basis of actual evidence; or c) because it is an idealization and, as such, cannot be seen as applicable to any actual set of objects (Mäki Unrealistic assumptions and unnecessary confusions, Ng Are unrealistic assumptions/simplifications acceptable?). Although we can agree that this distinction is crucial for a rigorous defence of the irrelevance thesis, for the present purposes it is not. I will simply accept it as sound in its general form, having in mind that the notion of assumption is understood mainly in a logical sense. The acceptance of this thesis is important because it supports the positivist idea that a theory should be evaluated based on its empirical contrastability. Some assumptions may not be true regarding the actual, empirical world. But taking them into account may help to contrast a theory with the actual, empirical world. However, as I would like to show, the irrelevance thesis is not enough for an appropriate positivistic account of economics, having in mind the general formulation of positivism proposed above (2.6). 


\section{Economic growth}

After pointing out some notions related to economic positivism and positivism in general, I will, in this and in the following sections, get into some philosophical problems related to the concept of growth and to the foundations of macroeconomics. The notion of growth is one of the main topics of neoclassical macroeconomics. Its relevance is associated with the ways in which it relates normative and positive economics. As already mentioned, and as should become clearer later, the relation between both will vary depending on the economic approach that one adopts.

Friedman's thesis about the irrelevance of unrealistic assumptions did not have as much direct influence on economic theory as on economic methodology. However, his thesis of irrelevance mirrors very faithfully neoclassical positivist perspectives on growth. Friedman's direct contributions to economic theory were mainly on monetary policy, inflation, and employment. Of course, these areas are linked to economic growth, but indirectly. Anyway, a study of Friedman's viewpoints on growth is beyond the scope of this work. Rather, I will consider some epistemological implications of the irrelevance thesis and suggest ways to tackle certain issues associated with it and with neoclassical economic growth in general.

Mainstream models of economic growth are the ones developed by the neoclassical school and are based on a function of the following general form (Solow Technical change and the aggregate production function):

\section{(3.1) Production function $\quad Y=f(K, L)$}

Here, $Y$ represents output, $K$ represents quantity of capital, $L$ represents labour and $f$ is a production function. It is important to mention that $f$ should satisfy the condition of diminishing marginal returns, which can be characterized as follows:

(3.2) Diminishing marginal returns. For an agent, the marginal utility of a good decreases when the quantity of that good increases.

I will not put special focus on this condition now. It should be mentioned, however, that it also takes the form of a key principle in microeconomics, particularly in price theory (Marshall Principles of economics). As will be clear later, the notion of price grounds one of the main conflicts within neoclassical economic theory.

Considering economic growth, let us keep in mind the positivist idea that a good theory must have high predictive power about a certain class of phenomena. Whether or not its assumptions are realistic remains irrelevant (2.3). Under this principle, the definitions of the concepts of capital and labour, as well as the relations between them, are not important for the explanation provided by the production function. And neoclassical economists seem to have a clear explanandum in mind, namely, growth. How do we explain that some economies grow more or faster than others? All models based on the structure presented in (3.1) assume that capital and labour are main factors that should be involved in any explanans motivated by questions of that kind. But what is capital? And what is labour? According to the positivist perspective characterized above, it does not really matter. In principle, we could construct a good theory of economic growth based on poorly defined 
or unrealistic concepts of capital and labour. If the theory has acceptable predictive power, unrealistic assumptions and definitions should be harmless.

Here is, again, the main idea of Friedman's argument: theoretical assumptions can be considered as premises in a more general, deductive structure, and, thus, as antecedents of propositional conditionals. So, just as the truth of an antecedent does not guarantee the truth of the consequent, the truth of the assumptions within an economic theory is irrelevant for the correctness of its predictions.

The thesis for the irrelevance of assumptions (2.3) is, in certain terms, plausible, although Friedman's argument to support it lacks clarity at some places and may be regarded as inconclusive. As Ernest Nagel argues (Assumptions in economic theory), Friedman is not clear enough about crucial notions such as the notion of theory or the notion of unrealistic assumption. A somehow similar observation is made by Uskali Mäki (Unrealistic assumptions and unnecessary confusions), who argues that Friedman mixes and confounds concepts and perspectives that should be considered carefully for the sake of a better argumentation regarding economic issues. Developing another kind of criticism, Daniel Hausman (Why look under the hood?) claims that the realism of assumptions is important for a broader understanding of economics.

Since I am now interested in the critical analysis of economic growth, it seems appropriate to focus on the ways in which instances of growth models can be reformulated as propositional arguments. After doing this, a second step will be to consider the soundness of these arguments. It can be shown, as we will see, that one of the main possibly vicious argumentative features that one can associate with neoclassical growth models is their circularity (Robinson The production function and the theory of capital, Cohen \& Harcourt Retrospectives: whatever happened to the Cambridge capital theory controversies?). I will argue that such a circularity provides good reasons to criticize models of economic growth without rejecting the irrelevance of assumptions thesis. In a certain sense, I will criticize a part of mainstream positive economics from a positivist perspective (or a perspective that remains as positivist as possible). In this paper (sections 5, 6 \& 7), I will suggest that a fruitful way to tackle the issue of circularity can be found by focusing on the microfoundations problem.

\section{The problem of circularity}

A widely discussed problem of neoclassical growth models is the issue of circularity. The issue can be characterized as follows (Cohen \& Harcourt Retrospectives: whatever happened to the Cambridge capital theory controversies?, Kincaid Explaining growth):

\section{(4.1) Circularity of growth models}

4.1.1. To obtain a production function for (an aggregate of) diverse goods, outputs and inputs cannot be measured in physical terms.

4.1.2. They must be measured in value.

4.1.3. To have a value measure for diverse goods implies that there is a price for capital and for a distribution of income.

4.1.4. The price of capital (an input of the production function) depends on its productivity. 
To grasp the circularity more clearly, we must focus on price theory (Marshall Principles of economics). Very roughly, neoclassical price theory is based on supply and demand as main conditions, such that the following holds:

(4.2) Price. Under certain fixed conditions, the price of a particular good increases with the increase of demand and decreases with the increase of supply. Also, given a fixed price, the relation between supply and demand of a particular good reaches an equilibrium at some point.

So, here is the circularity. The explanation of a given outcome depends on valuations of capital and labour, but, at the same time, those valuations depend on what the factors can produce. As Alfred Marshall claims, "the chief demand for capital arises from its productiveness" (2013: 68).

Just for the sake of illustrating the circularity problem, consider this proposition, which can appear as a prediction obtained from different growth models under specified conditions:

\section{(4.3) China's economy grew by $6.9 \%$ in 2017}

It may be noted, before moving forward in the argument, that this should be taken as part of a general exemplification of the theoretical issue under consideration (the problem of circularity) and not as a particular case under study. The main point here is not about China's economy, of course.

Again, since I am trying to stay within a positivist perspective, I accept that it does not matter whether a description like 4.3 is realistic or not for the evaluation of neoclassical growth theory. By doing this, the reference of "China's economy" can be any entity or set of entities postulated in the modelling process. If the model requires to think of China's economy as a computational data set, let that be. The main question here is how we should understand a predicate like "grew by $6.9 \%$ ". Let $Y_{1}$ and $Y_{2}$ represent China's total production in 2016 and in 2017 respectively. Thus, we can reformulate 4.3 as follows:

\section{(4.4) The percentage ratio between $Y_{1}$ and $Y_{2}$ is $6.9 \%$}

Now, both $Y_{1}$ and $Y_{2}$ should be defined based on the general structure of production functions in terms of capital and labour (3.1). The sense of $Y_{1}$ as well as the sense of $Y_{2}$ is clear. But as stated in the second premise of the circularity problem (4.1), to obtain $Y_{1}$ or $Y_{2}$ we need to measure them in values. The same holds for the corresponding inputs. This means that there are expressions used in the theory whose denotations are obscurely determinable.

Let us just focus on $Y_{2}$ and symbolize China's invested capital and labour during 2017 as $K_{2}$ and $L_{2}$ respectively. And let lowercase letters represent the values taken by each variable, such that the expression of the form $A=a$ symbolizes the fact that $A$ takes the value $a$. Thus, considering growth theory, we arrive at the following:

(4.4) $K_{2}=k_{2}$ (together with $L_{2}=I_{2}$ ) explains $Y_{2}=y_{2}$

Now, based on price theory, we could also claim this:

(4.5) $Y_{2}=y_{2}$ explains $K_{2}=k_{2}$ 
This should make the circularity problem clearer. Considering 4.4 and 4.5 , the values of $K$ both explain and are explained by the values of $Y$. The positivist concepts of capital, production, labour, and growth remain without a clear definition, even when conceptual clarity is one of the aims of positivism (2.6.3). Note that the issue arises within the framework of positive economics and, therefore, respecting the irrelevance of assumptions principle. The recalcitrant question for the defenders of the neoclassical program, then, is whether their methodological positivist principles deserve to be considered as constituting a good example of positivism.

We should also note that this issue can be generalized as a criticism against reductionist empiricism in general. Any positivist account according to which some strict notion of experience is supposed to be the only crucial methodological basis for the evaluation of theories may have problems of circularity like the ones indicated in this section. This does not mean, however, that positivism is a completely nonsensical or contradictory approach that presupposes, thus, an impossible discourse. As we will show, a more open empiricist perspective can be very fruitful.

\section{The problem of microfoundations}

As shown in the last section, there are predictions obtained from neoclassical growth models whose content can only be determined, on pain of circularity, if certain variables are also assumed in some respects as explananda of what they initially tend to explain. In this way, we have reasons to criticize a part of positivist economic theory from a positivist stance, i.e., considering its empirical consequences rather than the plausibility of its assumptions. Note that my argument is not mainly about the theory-ladenness of experiments, but about a special theoretical and conceptual circularity within mainstream economics. How this circularity may depend on some general theoryladenness could be the topic for another work, but it is not part of the main issue here.

Note also that what we can consider as a negative aspect of some economic growth models is not about the deducibility of their predictions, but about the circularity associated with them. Now, is this a vicious circularity? Which features should a good economic theory have to tackle the problem of circularity? I will propose some ways to approach these questions in what follows.

As pointed out, price theory is crucial to understand the circularity problem related to growth: capital and labour explain production, but production explains the values (prices) of capital and labour. Now, it should be emphasized that this is not a tension within economic growth theory in isolation, but a tension between macroeconomics and microeconomics. On the one hand, price theory seeks to account for the behaviour of individual rational agents and is thus part of microeconomics. On the other hand, economic growth theory accounts for the behaviour of economies and is, as such, part of macroeconomics. Considering this, I think that the problem of circularity should be tackled having in mind the theoretical and epistemological relations between micro and macroeconomics. The analysis of these relations leads us immediately to the so-called problem of microfoundations, which can be characterized as follows (King The microfoundations delusion, Pilkington The reformation in economics):

Microfoundations problem. How do the actions of individual rational agents determine high-level properties of an economy that they constitute, considering that these macro properties also determine the actions of individuals? 
The first part of the issue can be considered from two angles: a) In which sense are macroeconomic processes, such as growth, reducible to collective behaviour at the low-level? b) How do macro processes arise from the latter? The second part of the problem can be expressed as a problem of downward causation, i.e., in which way can we characterize that high-level economic states have causal power over the behaviour of individual agents?

Considering these aspects of the microfoundations problem, it seems more than appropriate to approach it in the light of the notion of emergent property. Very roughly, the property of a system is emergent if it arises from the complex interactions of the system's constituents but is at the same time irreducible to them (Broad The mind and its place in nature, Alexander Space, time and deity, El-Hani \& Pereira Higher-level descriptions).

The next sections will be focused on the following. First, I will consider a characterization of emergence that may allow us to tackle the microfoundations problem. Then, I conjecture that there is a promissory way of clarifying, on this basis, the circularity problem of growth theory.

\section{Contextual emergence}

The rough characterization of emergence given at the end of the last section involves a conundrum in its very basis: how can a property be determined and undetermined at the same time by the constituents of the system in which it arises? While reductionists dismiss the notion of emergence trying to explain how macro properties can be determined by micro properties, dualists do it proposing that some macro properties are irreducible in some absolute sense. I would like to explore the possibility that emergent properties are indeed reducible and irreducible at the same time. But, of course, as it stands, this is a contradiction. However, the notion of an epistemic context, mentioned earlier, allows us to escape incoherence:

(6.1) Epistemic context. An epistemic context is a structure $\langle Q, R>$, where $Q$ is a set of (descriptive, normative and/or nominative) expressions, ordered by a set of relevance functions $R$.

Epistemic contexts are given within the course of some investigation and, thus, are ways of representing pragmatic aspects of contingent, human endeavours. This justifies the inclusion of normative and nominative expressions besides descriptions. In general, investigation does not only involve descriptions, i.e., positive items, but also attitudes associated with how we approach phenomena, how we attend to objects and how we evaluate them. It is usual to find out that norms, attitudes, and phenomena are very hard to measure, quantify or translate to a positive language. They often oscillate between the inaccessible and the arbitrary. And we might agree, but this does not mean that considerations about non-descriptive items must be eliminated from theory, as strict economic positivists seem to promote.

Now we may characterize the notion of an emergent property as follows, where $K$ is a general epistemic context from which we can refer to a set of contexts $C_{i}$ that we may call sub-contexts:

(6.2) Contextual emergence. According to a context $K$, a set of states, symbolized by $E$, is emergent from another set of states, symbolized as $B$, just in case

6.2.1. there is a context $C_{1}$ in which $E$ is reduced to $B$,

6.2.2. there is a context $C_{2}$ in which $E$ is irreducible to $B$ and 
6.2.3. there is a context $C_{3}$, according to which $E$ involves observed, novel properties that are correlated with an abrupt change of complexity involved in $B$.

On this basis, we can also understand an emergent process as a process in which a certain change of complexity occurs, associated with a corresponding emergent property, and an emergent state as a state that involves an emergent property. Note that this definition of emergence is not only applicable to issues regarding the relations between micro and macroeconomic phenomena, but to any complex system (or set of complex systems) in which high-level properties are hard to explain or to predict based on its low-level properties.

The notion of complexity, included in condition 6.2.3, is crucial if we want to require an understanding of emergent properties not as mere bogus, phantasmagory properties, but as properties associated with physical ones and with structural changes at the physical level. Complexity also expresses the point that emergent processes arise from numerous and diverse interactions. Somehow, it keeps us away from dualism. Emergent properties are neither just put there nor appear as completely separated entities. This is particularly important about economics. Is capital, for instance, just something that we can aggregate to a system? Or is it rather something that forms within a system, given various changes related to the different complex processes that constitute it? The notion of emergence motivates us to put more emphasis on the second question. However, note that it does not force us to reject the first as unimportant. According to the characterization just proposed, emergent properties not only can be understood reductively, but also must be understood in this way. This does not imply a surrender to reductionism. Although the account accepts reductions, they do not count as the main relations that explain emergence.

It is worth to recall that John Stuart Mill (A system of logic) developed one of the first notions close to emergence, explaining that some effects of organized bodies are fundamentally different from the effects produced by their components. Thus, it is more than plausible to think that he assumed this kind of relation when he claimed, for instance, that capital arises from accumulation or that profit arises from the productive power of labour (Mill Principles of political economy). Furthermore, it is crucial to consider how these ideas influenced Karl Marx's theory (Balassa Karl Marx and John Stuart Mill, Shoul Similarities in the work of John Stuart Mill and Karl Marx, Evans John Stuart Mill and Karl Marx).

Considering context-dependence, the Fregean distinction between sense and reference may be important here (Frege Über Sinn und Bedeutung). The idea can be put in a quite simple way: the sense, i.e., the cognitive or theoretical meaning of $E$ varies from context to context. And its reference, namely its ontological meaning, is determined (or postulated) depending on each context. This allows us to refer one single expression to different, sometimes incompatible ontologies.

The relation between two contexts $K$ and $C$, where $C$ is a sub-context of $K$, is not extensional, but intensional. This means that $C$ is not necessarily a subset of $K$. However, it is important that we can mean something about $C$ from the perspective of $K$. In other words, some expressions of $K$ may have their reference in $C$, while their sense may be determined depending on the role each expression plays within $K$ itself. This allows us to consider expressions for an emergent state $(E)$ within different contexts and, at the same time, relating those expressions inter-contextually. For instance, we may consider a neoclassical, reductionistic sense for the expression "capital" within some context $C_{1}$ and 
a non-reductionistic sense, within a different context $C_{2}$, characterizing a classical notion focused on the organic aspects of capital. Both contexts may i) refer to each other, ii) be associated as subcontexts with a third context $K$ and even iii) refer together to a same set of entities within a postulated ontology (say, a sociological ontology). These kinds of relations are crucial to understand certain economic states as emergent, according to my proposal.

Essentially, the contextualist strategy applied here can be considered as part of a broader framework, which includes, among others, epistemic and linguistic versions of contextualism (DeRose The case for contextualism, Kompa Contextualism in the philosophy of language, Bouchard Epistemic contexts and indexicality). Thus, the general form of the present argument does not hang necessarily upon the specific cases regarding economics studied in this work.

Furthermore, reference relations allow us to characterize the reflexivity of economic systems in terms of self-reference. An economic system $S$ can be seen as reflexive when it can be represented as a general context $K$, based on which we can refer to another context $K^{\prime}$ that represents $S$ in a different sense. I will not focus on the notion of reflexivity in more detail here (Gay Reflexivity and development economics, Schliesser Four species of reflexivity and history of economics in economic policy science, Davis \& Hands Reflexivity and economics). However, it may settle a ground to argue in favour of the relevance of assumptions. An economic assumption can be treated as realistic than another depending on how they are projected to the ontologies of the most general and selfreferential economic contexts. For example, even if assumptions regarding fully rational agents may work on restricted contexts, they may or may not be projected to the ontologies of a more general, self-referential economic system. And either way will have important repercussions on how the system develops. The unrealisticness of assumptions is important in this sense.

It should be mentioned that the present proposal can be considered as an account concerning scientific inquiry in general, such that my argument about taking different contexts simultaneously does not depend necessarily on the issues of microfoundations and circularity associated with economic growth theory. Emergence can be described about different levels of explanation, not only to the social level. Various related notions of contextual emergence have been applied to physical, biological and cognitive processes (Atmanspacher Contextual emergence of mental states, Fuentes Complexity and the emergence of physical properties, Van Gulick Reduction, emergence and other recent options on the mind/body problem).

Now that I have proposed a framework, in the next session I will discuss how it may be applied to tackle the problems already characterized, without falling in vicious explanatory circularities and staying closer to what positivism should be (2.6) than what neoclassical theorists seem to think it is.

\section{Discussion: microfoundations, aggregation, and emergent macroproperties}

It is no surprise that defenders of the neoclassical doctrine try to account for the microfoundations problem in a reductive manner, considering that their production functions operate over variables that simply acquire their values depending on microeconomic processes regarding price. These are some of the common assumptions that pave the way for this task, concerning economic growth theory in particular (Hoover Reductionism in economics, Shaikh Capitalism: competition, conflict, crises): 
a) Hyperrationality. All agents act maximizing their utilities and based on perfect information.

b) Agent aggregation. Given hyperrationality, we may add the activity of all firms, consumers, and workers to treat them as a single variable (for example, labour as input for a production function).

c) Homogeneity of capital. All items considered as capital can be treated as homogeneous thanks to their valuation based on price theory.

d) Capital aggregation. Given the homogeneity of capital, we can treat all capital as a single variable.

Based on these assumptions, some macro properties are just aggregates of micro properties. Now, while emergent properties are usually understood as properties that are more than the sum of its parts, it seems plausible (if not obvious) to take any neoclassical strategy based on aggregation as an attempt of treating macroeconomic properties as mere sums of their parts.

The microfoundations program based on the mentioned assumptions does not seek to provide explanations of how economic high-level features (such as growth) emerge from interactions at the low level. Given the relevance of price theory within neoclassical theory, the aim of providing microfoundations is rather associated with considering aggregation issues as serious and tackling them in ways that may tend to eliminate macroeconomic terms in favour of an economic theory based on what we now understand as microeconomics (Lucas Models of business cycles). Note how this reductionist stance is at odds with the condition of scientific unity, proposed earlier for a general account of positivism (2.6.4).

As explained earlier, neoclassical growth theory implies an explanatory circularity about the notion of production and its valuation. Roughly, the problem can be considered in the light of this question: Do supply and demand explain growth or does growth (in particular, profitability) explain supply and demand?

Capital and labour get their values through supply and demand relations, described by the theory of price, and having certain specific values, they explain growth, under a given production function. But for the same reason, i.e., since the very valuations of capital and labour depend on (and are explained by) their expected profitability, they also depend on production, more precisely on pricerelated expectations thereof.

Again, is this a vicious circularity? It would not have to be, if we adopted the reductionist neoclassical program. In a radical reductionist perspective, capital, labour, and production are just epiphenomena. They do not have the "real" causal power with which we could explain economic processes, because every economic process operates as a set of relations based on supply, demand, and optimization. So, according to this view, there is no vicious circularity because there is no circularity at all.

It would be a vicious circularity, however, if we adopted a dualist perspective, i.e., a view that maintained micro and macroeconomic processes as separated as possible. This also seems to be the stance of mainstream economics sometimes. But when economists depart from dualism and accept that microeconomics and macroeconomics are focused on the same system of phenomena, they come to what we understand as emergence. As an example of this, let us consider one of Paul Krugman's contributions. Krugman has made a great effort to understand economies as irreducible complex systems. In this regard, he introduces the notion of spatial economy, which can be characterized as follows (Krugman The self-organizing economy): 
7.1 Spatial economy. A spatial economy is a system constituted by agents (firms, for example) to which we attribute certain states, namely locations at given times. These components interact in multiple ways, depending on needs and resources. Interesting patterns of self-organization arise from these interactions.

Note that from this perspective, we do not have to deny that production depends on capital and labour. We could explain in a reductive manner, through some production function, why a given economy is growing at a certain rate, aggregating the labour and capital power associated with the set of agents that constitute the system. For that matter, we could obviate their locations. However, by considering the spatial features of an economy we can also explain processes such as the concentration and mobility of factors. These processes are crucial for an understanding of production, capital and labour that is undoubtedly richer than reductionist alternatives.

We can think of Krugman's notion of spatial economy as a case that supports the characterization of emergence given earlier. Take again, for example, the observation that China's economy grew by 6.9\% in 2017. Following definition 6.2, we can express that China's growth is an emergent process that arises from the interactions and motions of its components, as follows:

7.2. China's growth as an emergent process. First, we have reductive contexts that seek to explain that phenomenon. Second, we have non-reductive contexts that may only point to rough relations between the phenomenon and the interactions at the micro-level or according to which China's growth may seem surprising. And third, we have epistemic contexts in which abrupt fluctuations of complexity may indicate correlation patterns between China's growth and China seen as a spatial economy.

It is important to keep in mind that this proposal is not strange to positivism. It is data-based, it is explanatory, it allows for simple predictive models, and it is even compatible with Friedman's principle of irrelevance: whether the ontologies that one may postulate based on a reductive context are idealizations or too abstract or plainly false does not matter. But we must add it does not matter if we also take other contexts into account based on which we may arrive at a broader understanding of economics. This is very far from Friedman's view of positive economics.

We cannot just say that the realism of the assumptions is irrelevant if they support models with acceptable predictive and explanatory power. We must consider other epistemic criteria, such as the comprehensibility of an economic model, its relation to models of other disciplines and even the possible ethical (not just explanatory) consequences of their applications.

The main problem with Friedman's positivism that I want to point out is not about its plausibility, but about its methodological narrowness. According to Friedman, economics is only about events, not about evaluation (The methodology of positive economics; Value judgments in economics). By contrast, the concept of epistemic context that I assume here involves not only descriptions and explanations of events, but also normative statements of different sorts.

Let $K$ be an epistemic context from the perspective of which we assess some economic state $E$ as emergent. Recall that, according to the definition proposed here, there must be three sub-contexts to which we may refer from $K$ and based on which we may explain $E$ in different ways. Context $K$ may involve different normative and methodological criteria that may drive the selection of those 
sub-contexts. Should we not include ethical and social criteria as well to do that? The answer is simple: we should include them, given the nature of the discipline and the types of phenomena it seeks to explain and control. But what about physics? How do they manage to offer their highlyexplanatory models without focusing much on ethics? (An orthodox may insist). Well, if the phenomena and the relevant context do not require it, ethical considerations may be negligible. Perhaps there is no big issue in giving a reductionistic explanation of motion at some scale to explain certain macroscopic phenomena, such as sea levels, for instance, mainly considering gravitational and rotational forces. But, again, nothing stops us from considering other factors, such as pollution. And this is how contexts in which things turn normative gain relevance.

The positivistic features of economics, i.e., its empirical aspects, can be characterized following Otto Neurath's view on scientific progress in economics (Nationalökonomie und Wertlehre):

7.3 Economic progress. Progress of economic theory is possible when abstractions are obtained from empirical complexes, which can be combined and lead to further investigations. What we render as empirical is a matter of principle.

These aspects of scientific progress can be found, of course, in other areas. And I would like to put emphasis on the idea that the notions of epistemic context and contextual emergence could be applied not only within scientific fields, to establish relations between separate research projects, but also to consider inter-theoretic and inter-disciplinary relations. And it is also important to emphasize that contextual aspects of progress are fundamental in economics. In 1910 Neurath understood such aspects as follows: "[I]t is possible to leave measurable quantities behind and yet do exact science. Since in political economy we are able to symbolically represent systems of organisations it might also be possible that other branches of the so-called social sciences are amenable to exact treatment. This would be a large step towards the creation of a universal science. It is a major task to render the whole order of life as transparent as possible and to reduce as many relations as possible to simpler ones. [...] The biggest difficulty consists in isolating the separate investigations as far as possible without losing perspective on the other contexts. One has to be aware at all times whether it is appropriate to retain the entire system of the theory and account for some fact by auxiliary hypotheses or whether it is more appropriate to rearrange the entire system. This is not always strictly adhered to. All too often the separate sciences forget the larger contexts and go beyond their field of competence by drawing conclusions about the whole state and the entire social order from a small number of premises that had been established for just a few problems. On the other hand, it is not rare that in discussing a certain concrete context absolutely vital circumstances are not examined and that instead general ideas are invoked in some vague way (the rhythm of social life etc.). This phenomenon is not only observable in political economy but also, for instance, in probability theory, occasionally in biology and in other places too" (Neurath 2004:286).

Data obtained from price fluctuations can be considered as empirical from a neoclassical, and even from a contextualist perspective. But they can also be part of an empirical complex, constructed from observations of different sorts, abstractions, and combinations thereof. Thus, going back to our example, the fact that China's economy is growing fast is, as a matter of principle, empirical. Various interests and methodological aspects shape these abstractions, their combinations and how we describe them. To ignore this implies not only ignoring the potentials of economic theory, but also ignoring crucial aspects of empiricism and positive science. 


\section{Conclusion}

It has been argued that economic growth may be better understood based on the notion of emergence than based on the traditional, neoclassical perspective. According to such a perspective, growth is characterized in terms of output ratios associated with a production function. This functional analysis usually depends on assumptions that may seem plainly unrealistic, e.g., the assumption that economies are closed systems or that capital and labour are independent. Rather than criticizing this kind of models for being unrealistic, one can criticize them for implying a certain circularity (section 4). As shown, the problem of circularity is an aspect of the vastly debated microfoundations problem (section 5). To tackle this problem, it is here suggested that, instead of adopting a simple form of reductionist positivism or of dualism (which may also lead to a lot of unclarities), we can focus on an emergentist view based on the notion of epistemic context. As pointed out, this strategy is compatible with traditional positivist principles about the relevance of experience and explanation of facts. However, it goes further, involving considerations about normativity, complexity and even about what turns out to be unexplainable within a particular economic inquiry.

The present proposal implies three main contributions to the philosophy of economics. (I would like to thank an anonymous referee for emphasizing on these consequences of the proposal and for helping me formulate them in a clear way, considering their impact within the philosophy of economics.) First, I move the focus of the debate about positive economics from considerations of theories seen in isolation to considerations of interrelated projects of inquiry, trying to argue for an analytical frame in which epistemic aspects of individual theories and research projects must be assessed in reference to wider sets of ongoing research projects. This first contribution can also be seen as an argument about the importance of the collective aspects of scientific research, inviting us to reconsider the concept of explanation as well as the role that individuals play within a wider, ongoing system of collective inquiry.

Second, and more specifically, I argue that local considerations of validity, which have been traditionally central in debates about positive economics, can be loosened by moving from a focus on individual theories to considerations of families of interrelated theories and models. So, how realistic are the assumptions of a given economic model may not always matter. But we should have always in mind the explanatory, normative, and observational restrictions of the epistemic context in which they operate. Irrelevance of assumptions may be valid in its contextually limited methodological domain if we also consider a pluralistic and broad view on economics.

And third, I think that the present proposal significantly moves the discussion about methodology in the philosophy of economics forward, where simplistic debates about methodological collectivism and methodological individualism seem perennial. I suggest a novel and productive way of integrating both projects, which allows us to retain the acceptance of their contributions, limitations, and autonomy.

The second and third contributions, which imply important advancements in our understanding of multi-level economic phenomena, promote a pluralistic and open, positivist philosophical spirit, a spirit that is perhaps needed today more than ever in economics. Having this notion of epistemic openness in mind, the specific aspects of the philosophy of economics approached in this work are also particularly relevant in the sense that they help us make an argument about the nature of 
science and social epistemology in general. The pluralistic aspects implied by my perspective are, in certain relevant ways, harmonizable with the account proposed by Victoria Chick and Sheila Dow (The meaning of open systems), which is based on the concept of an open formal system.

Some crucial issues remain, though. How should openness be regulated? Institutional restrictions depend on networks of power relations, based on which material and theoretical developments are fixed and transformed. So, the ways in which economic processes are described according to a mainstream are themselves determined by economic processes. (But does the term "economic process" preserve its meaning in this last sentence?). How should we interpret the idea of coherence in condition 2.6.4 then? It seems that it cannot be a simple, classical, first order coherence, if we admit that social processes always involve struggle and clashes of different forces. If our main interests are focused on life and self-organized coexistence between different individuals and species at a global level, then our notion of coherence should accommodate to those interests. And some notions, like the notion of a rational agent, for example, may not be fundamental there, i.e., they may not constitute a basis for emergence relations that are deemed relevant in that context. A very different case is where interests are focused on control, exploitation of the environment and accumulation of wealth. Not only a different notion of coherence will set the structure according to such a context, but it will be at some point an imposed coherence as well.

The idea of emergence that I defend in this work is flexible enough to distinguish between those different types of contexts. It is not restricted, like other concepts of emergence, to the domain of the natural sciences, because a lot will depend on how far we are able (or allowed) to generalize and on how sensitive we can be about our interests and of others. Considering all this, it may be questioned whether being positivist is a characteristic of my criticism at all. Well, it may not be positivist in the dogmatic sense, but the proposal still demands strong conditions regarding empirical adequacy and logical analysis. It is not that a positivist stance is strictly required to solve the problems of circularity and of microfoundations, but it is a stance that could be chosen to confront them. Anyhow, the correct positive stance to tackle them is not what neoclassical economists call "positive economics", as I wanted to argue. So-called positive economics is a stance that rather ignores and avoids those problems. Thus, my proposal is a way to recall that reductionist positivism and positivist economics fall short in grasping important notions and tackling some crucial philosophical problems in the social sciences. As I tried to show, this can be done by instead seeking for the radical openness and diversity of epistemic systems.

\section{Acknowledgements}

The production of this work has benefited from discussions at Buenos Aires University, Santiago University and Santa María University. I would like to thank Sheila Dow, Ronald Durán, Raúl Fuentes, Javier Legris, and Oscar Orellana for valuable conversations, as well as to the Chilean Agency of Research and Development for financial support (projects \#11180624 \& \#1181414). 


\section{References}

Feigl, H. (1943). Logical empiricism, pp. 371-416. In D. Runes (ed.). Twentieth century philosophy. Philosophical Library. https://archive.org/details/in.ernet.dli.2015.25045

Marshall, A. (2013). Principles of economics. Palgrave Macmillan.

Neurath, O. (2004). On the theory of social science, pp. 265-291. In T. Uebel and R. Cohen. Economic writings selections 1904-1945. Springer. https://link.springer.com/book/10.1007/1-4020-2274-3

Received 18 Jun 2021

Accepted 3 Sep 2021 